\title{
De la compuerta a la neuromatriz y neuromodulación
}

\author{
From gate and neuromodulation to neuromatrix
}

Andrés Rocha', Ángel Juárez', Giancarlo Ferretiz

\begin{abstract}
The following article presents a review of the evolution of the gate and neuromatrix theories as well as their contribution in the development of neuromodulation to the present day. Nowadays the mechanism of action of neuromodulation is better to understand by dividing it according to the level where it acts (local, central or peripheral). Patient selection is crucial to optimize the effectiveness of the device; although the indications for pain that support current guidelines are failed back syndrome, complex regional pain syndrome and lower limb ischemic pain and refractory angina; neuromodulation is already being extended to multiple fields of action that affect the future, such as the improvement of human capacities.
\end{abstract}

\section{RESUMEN}

El siguiente artículo consiste en una revisión no sistemática de la evolución de la teoría de la compuerta y la neuromatriz, así como su aporte en el desarrollo de los dispositivos de neuromodulación. Para entender mejor el mecanismo de acción de la neuromodulación se divide según el nivel en que actúe sea local, central o periférico. La selección de pacientes es crucial para optimizar la eficacia del dispositivo; aunque las indicaciones para manejo del dolor que soportan las guías actuales son síndrome de espalda fallida, síndrome doloroso regional complejo y dolor tipo isquémico en miembros inferiores y dolor por angina refractaria; actualmente la neuromodulación se está extendiendo a múltiples campos de acción entre los cuales se encuentra el mejoramiento de las capacidades humanas.

\section{Key words:}

Gate theory, neuromatrix, neuromodulation

\section{Palabras clave:}

Teoría de la compuerta, neuromatriz, neuromodulación

1 Clínica del Dolor Instituto Nacional de Cancerología, DF Ciudad de México.

Fecha de ingreso: 22 de febrero de 2019

Fecha de aceptación: 14 de abril de 2019

\section{ORCID}

https://orcid.org/0000-0001-6860-8774

Correspondencia:

Dr. Andrés Rocha.

Email: rocharomeroandres@me.com 


\section{Introducción}

E I avance de las neurociencias ha dado pasos gigantes en las últimas décadas, esto gracias al desarrollo de nuevas técnicas de monitoreo invasivo y no invasivo que permiten ver la actividad del cerebro in vivo. En la actualidad, se gestan proyectos ambiciosos tales como BRAIN (Brain Research Through Advancing Innovative Neurotechnologies) en Norteamérica y el proyecto Cerebro Humano de la Unión Europea[1].

La Asociación Internacional para el Estudio del Dolor (IASP por sus siglas en inglés) nos define el dolor como una experiencia sensorial o emocional desagradable asociada a un daño real o potencial en un tejido, o descrito en términos de dicho daño. La construcción de esta definición no ha sido fácil y ha involucrado amplia experimentación y formulación teórica; esta conceptualización abre el campo al llamado dolor total ya que no se define solamente por sus propiedades biológicas, sino por conceptos psicológicos y sociales[2].

\section{Sobre el Dr. Ronald Melzack y el Dr. Patrick David Wall}

Dentro de los pioneros en el campo de la investigación del dolor pocos han llegado a tener tanta fama como el Dr. Ronald Melzack y Dr. Patrick David Wall.

El Dr. Patrick David Wall nació el 25 de abril de 1925 en Nothingham en la zona central del Reino Unido, en el seno de una familia acomodada de la época. Su padre era profesor de la Universidad de Middlesex. Uno de los acontecimientos que lo marcó fue a los 10 años en que sufre de una hernia inguinal estrangulada que requirió múltiples tratamientos obteniendo un inadecuado control de su dolor. Cuando cursaba en la escuela de San Pablo en Londres estalla la Segunda Guerra Mundial lo cual motivó la migración de muchos académicos, en ese momento queda como director académico un recién graduado en zoología, Prof. S.A. Barnett (por problemas de salud no pudo enlistarse en el ejército). Posteriormente, ingresaría a la Universidad de Oxford que una vez pasada la guerra estaría llena de figuras como el Prof. Sir. Charles Scott Sherrington (Nobel de Medicina 1932 por sus trabajos en localización de las funciones en la corteza cerebral), donde se incorporaría a la investigación en el laboratorio de Alexander Fleming y sería uno de los fundadores del British Medical Student's Journal. A la corta edad de 21 años ya contaría con publicaciones en revistas como Brain y Nature, y en 1948 obtendría el diploma de especialista en Neurología. Sus primeros años de vida laboral los dedicaría a la atención de víctimas del holocausto y refugiados de Europa[3].

Años después migra a los Estados Unidos, incorporándose en 1950 a la Universidad de Yale e inicia su investigación sobre lobotomía (destrucción quirúrgica de las vías de conexión de los lóbulos cerebrales) como tratamiento de la depresión. Su vida académica fue fructífera ya que luego trabajo como profesor asistente en la Universidad de Chicago y más tarde en la Universidad de Harvard, terminando como profesor titular en el Instituto Tecnológico de Massachusetts en 1960 donde conocería a su compañero de academia el Dr. Melzack junto a quien publicaría quizás la primera aproximación a la teoría de la compuerta en 1962 en la revista Brain[4].

En 1967 el Dr. Wall publica, junto al Dr. Sweet, una serie de casos de 8 pacientes con dolor neuropático quienes reportaron alivio del dolor por más de 1 hora tras estimulación eléctrica[5]. Este artículo sería uno de los primeros trabajos en comprobar el efecto de compuerta y las aplicaciones primitivas de la neuromodulación.

Desafortunadamente sus problemas con el consumo del tabaco conllevan a que, en 1996 se le diagnosticara cáncer de próstata, posteriormente en 2001 tuvo una recaída tumoral requiriendo manejo quirurgico, pero falleciendo unos días después de su egreso.

El caso del Dr. Ronald Melzack no es el mismo, a diferencia del Dr. Wall, nace en el seno de una familia de escasos recursos el 19 de julio de 1929 en Montreal. Su padre mantenía a la familia con una librería. Por su aptitud académica fue elegido como el hijo en que se invertiría para que fuera a la Universidad. Realizó sus estudios en Psicología en la Universidad de Mc Gill bajo la tutela del Dr. O Hebb considerado uno de los padres de la Biopsicología con sus teorías sobre el ensamblaje neuronal; esta afirmaba que el cerebro elabora recuerdos intensificando los apareamientos entre las neuronas que se activan de forma concurrente lo cual sería comprobado años más tarde[6]. También se entrenó con Pavlov de quien aprende técnicas de experimentación en animales para estudiar la ingeniería del comportamiento.

Su principal interés eran los pacientes con algoalucinaciones, más específicamente dolor de miembro fantasma. También comienza a investigar sobre cómo medir o calificar el dolor y termina publicando junto al Dr. Torgerson el famoso cuestionario de dolor de Mc Gill[7], basado en una valoración multidimensional que expondría desde una triple perspectiva: Sensorial (descripción del dolor en términos témporo-espaciales); afectivo-motivacional (descripción del dolor en 
términos de tensión, temor y aspectos neurovegetativos) y evaluativo (dolor descrito en términos de valoración general) con 78 adjetivos distribuidos en 20 grupos[8].

Entre los principales aportes que realizó Melzack a la evolución científica del manejo del dolor, se encuentran la publicación junto a Wall de la teoría de la compuerta en el año 1965 y en el año 1999 la publicación de la Teoría de la Neuromatriz, donde integra y amplía lo desarrollado con la escala de dolor Mc Gill con una amplia explicación de las múltiples dimensiones del dolor[9].

Los aportes de ambos autores a la comprensión de los mecanismos del dolor por medio de la publicación de sus trabajos, son objeto del reconocimiento de la comunidad científica a nivel mundial con diversos premios; especialmente de los especialistas en el manejo del dolor, quienes a través de dichos aportes contribuyen día a día a mejorar la salud de los seres humanos que son objeto de estas intervenciones.

\section{Teoría de la compuerta}

La primera parte de la teoría fue descrita en 1962 en la revista Brain, no obstante, sería hasta 1965 cuando el Dr. Melzack y el Dr. Wall publican juntos la versión más completa de su teoría[10] por medio de la cual se integran los avances precedentes y desmitifican aspectos relativos a la comprensión de los mecanismos del dolor.

Para ese entonces existían al menos tres teorías predominantes que pretendían explicar el mecanismo del dolor, la teoría de la intensidad, la teoría de patrones y la teoría de la especificidad, entre las cuales había prevalecido esta última.

La teoría de la especificidad debe sus inicios a las descripciones hechas por Descartes[11]. Básicamente, la teoría dice que cada modalidad tiene un receptor específico asociado a una fibra específica; según la visión primitiva de Descartes (alrededor de 1662) expuesta en su "tratado del hombre", los nervios consistían en fibras únicas que se conectaban directamente al cerebro a modo de una cuerda que al ser estimulada vibraba y hacía sonar una campana que representaba la respuesta dolorosa, esta propuesta fue posteriormente acogida por Galeno y más tarde por Charles Bell, sin embargo, tomaría mayor fuerza con el descubrimiento de los receptores cutáneos (Pacini 1835, Meissner 1853, Merkel 1875 y Ruffini 1893) y la descripción de las 4 modalidades sensitivas propuestas por Max von Frey en 1894 (toque, calor, frío y dolor).

La teoría de la intensidad, también llamada de su- mación, se basaba en que si un determinado estímulo es capaz de superar determinado umbral se convertiría en doloroso simplemente por su intensidad sin importar que receptor estimulara. El mayor aporte a esta teoría fue el de Naunyn y Goldsheider a finales de 1800, en pacientes con Tabes Dorsal se aplicaban estímulos repetidos entre 60-600 veces/seg y después de cierto tiempo los mismos describían un dolor intenso, así Goldsheider propuso que los estímulos sensitivos convergen y se suman a nivel de la materia gris de la médula espinal generando dolor[11].

De forma paralela a lo anterior se desarrolló también la teoría del patrón, esta ignoraba por completo los aportes sobre receptores específicos y lo atribuía todo a los estímulos, no solo tomando en cuenta su intensidad, sino también el orden o patrón de disparo de los mismos, de manera que en cierta forma decodificaban a los receptores para activar modalidades específicas de respuesta.

Charles Scott Sherrington, en 1947, sería el responsable de unificar ambas teorías con toda la información recolectada hasta ese momento, de esta forma concluyó que si bien es cierto tenemos receptores que responden a un estímulo, la función de dicho receptor consiste en disminuir el umbral de excitación del arco reflejo para ciertos estímulos y elevarlo para otros; lo cual asumía por una parte la selectividad del receptor y por otro lado la intensidad del estímulo [4], [11].

En la descripción de su artículo en 1965 Melzack y Wall comienzan señalando las incongruencias de las teorías predominantes[10] según evidencia clínica, psicológica y fisiológica.

Para la evidencia clínica señalaron que se podía dar estímulo doloroso en ausencia de una conexión nerviosa, una estimulación de baja intensidad podía desencadenar un estímulo doloroso y más aún en ausencia de cualquier estímulo se podía obtener respuesta dolorosa. Ejemplo de lo anterior son los pacientes con dolor de miembro fantasma, dolor regional complejo y neuralgia periférica. Para la evidencia psicológica destacaron cómo un mismo estímulo genera diferentes respuestas según la personalidad y la situación de estrés en que se encuentre, así como los experimentos de condicionamiento de perros de $\mathrm{Pa}-$ vlov (al darles comida les aplicaba estímulo doloroso hasta que, posteriormente, evocaban sensación dolorosa con solo la comida). Para la evidencia anátomofisiológica se basaron en que dentro de las neuronas de segundo orden no solamente existen las neuronas nociceptivas específicas, sino las de amplio rango dinámico que responden no solo al dolor sino a muchos estímulos de bajo umbral, incluso hay neuronas no 
nociceptivas [4],[10].

Básicamente, la teoría de la compuerta, toma en cuenta las fibras delgadas sensitivas tanto las $C$ (cuya velocidad de conducción no supera los $2 \mathrm{~m} / \mathrm{s}$ ) como las A delta (cuya velocidad de conducción puede llegar a $20 \mathrm{~m} / \mathrm{s}$, pues cuentan con más mielina) y las fibras gruesas $\mathrm{A}$ beta que son motoras y actúan en músculos y articulaciones dando estímulos propioceptivos, cuentan con mucha más mielina por lo tanto, son más veloces y pueden llegar hasta $75 \mathrm{~m} / \mathrm{s}$. Ambas fibras siempre hacen relevo en la sustancia gelatinosa, en donde se encuentran interneuronas inhibitorias, antes de llegar a las células de transmisión que llevan el impulso hasta el cerebro; de modo que solo logra pasar un estímulo a la vez a través de esta sustancia gelatinosa la cual sirve de compuerta. Las fibras delgadas ejercen un efecto inhibidor sobre dicha compuerta de modo que las células inhibitorias ya no pueden ejercer su función de inhibir a las células de transmisión y así el impulso doloroso no se detiene y llega a generar percepción del dolor a nivel central. Mientras tanto las fibras gruesas tienden a activar las neuronas inhibitorias en la sustancia gelatinosa, de modo que la compuerta se activa dado que estas inhiben las células de transmisión y el impulso no asciende al cerebro[10].

La neuromodulación es mucho más compleja de lo descrito anteriormente, un ejemplo de esto se da con el dolor nociceptivo, donde la efectividad es muy pobre; además de que el alivio se mantiene por cierto tiempo aún después de apagar el neuroestimulador, lo que implica que no estamos actuando solamente en la compuerta[12].

\section{La teoría de la neuromatriz}

Según el mismo Dr. Melzack la contribución más importante de la teoría de compuerta fue el hincapié en los mecanismos del Sistema Nervioso Central. A partir de 1965 ningún especialista ha podido tratar de explicar el dolor en términos exclusivamente de factores periféricos. La nueva teoría obligó a las ciencias médicas y biológicas a aceptar al cerebro como un sistema activo que filtra, selecciona y modula los impulsos. Por otra parte, las astas dorsales, dejaron de ser estaciones de transmisión meramente pasivas para convertirse en lugares donde ocurrían actividades dinámicas: inhibición, excitación y modulación. En eso consistió la revolución: en que dimos protagonismo al sistema nervioso central como un componente esencial de los procesos nociceptivos.

El término neuromatriz fue propuesto por primera vez por Melzack en 1989[13], concepto que se consolidaría con su publicación en la revista PAIN en 1999. En su nueva teoría propone que en el cerebro existe una red neural del cuerpo en todas sus dimensiones que se encarga de integrar múltiples estímulos y generar un patrón de respuesta específica de dolor según corresponda. Para esto hay una relación entre las diferentes dimensiones del dolor y ciertas áreas del sistema nervioso central, entre estas se pueden citar el aspecto sensorial discriminativo, el afectivo emocional y el evaluativo cognitivo que se pueden correlacionar con la corteza somatosensorial, sistema límbico y componentes talamocorticales. En ese sentido se crea una repuesta neuroespecífica dependiente de la arquitectura sináptica codificada genéticamente en cada individuo, ejemplo de esto son los niños que al nacer lloran sin que nadie les enseñe, pero experimentan sufrimiento por el cambio brusco de entorno. Aunado a lo anterior, esta respuesta se va modificando según las experiencias que el individuo tenga a lo largo de la vida como lo son las situaciones de estrés físico o emocional en que se interactúa con el sistema neuroendocrino produciendo factores de inflamación.

A partir de lo anterior, Melzack explicaría diversas patologías[14] como lo son el dolor de miembro fantasma, en donde al perderse la modulación de los impulsos del miembro amputado la respuesta de la neuromatriz se expresa en ráfagas de alta frecuencia, características de la desaferentación y el dolor tipo calambre se da por la actividad neural que intenta activar los músculos perdidos, ambas respuestas se están originando en el cerebro y no en el sitio de lesión como tal. Otro ejemplo muy frecuente es la lumbalgia crónica conocida como dolor lumbar inespecífico ya que no se encuentra causa ni respuesta a los múltiples tratamientos, en este caso llama la atención la respuesta que tienen estos individuos a terapia complementaria psicológica, es decir, respuesta a nivel central.

La fibromialgia es un síndrome que se podría explicar como un estado de alerta que se mantiene de forma crónica ante un evento estresante por parte de la neuromatriz, lo cual genera fatiga y aumento en la sensibilidad ante cualquier estímulo. Por último, el autor explica el síndrome doloroso regional complejo como una enfermedad en la que el estrés juega un papel preponderante por encima del sistema adrenérgico, sobre todo en fases avanzadas de la enfermedad, lo cual explica que la respuesta al bloqueo con anestésico local disminuya con el tiempo[14].

Recientemente, lannetti et al.[15], contradice el concepto clásico de la neuromatriz sobre una relación específica por áreas del cerebro y describe que 
la respuesta se da por asignación de relevancia ante los estímulos, así la actividad cerebral está envuelta tanto en procesos nociceptivos como no nociceptivos, es decir, estas áreas detectan y reaccionan ante los estímulos produciendo un comportamiento apropiado ante el mismo.

\section{Historia de la neuromodulación}

Si bien es cierto la primera referencia a la palabra electricidad no fue usada hasta finales de 1500 por el científico inglés William Gilbert, ya se conocía el uso del pez torpedo en el tratamiento de la gota por Escribonio Largo, médico del siglo I en la corte del emperador romano Claudio. Para 1746, el físico Jean Jallabert, descubre que la actividad eléctrica se puede usar para estimular fibras nerviosas y lo usa en la extremidad de un paciente con parálisis de una de sus extremidades (para ese entonces ya se podía almacenar energía a través de botellas de Leiden), así observa no solo contracción sino regeneración del músculo y aumento del flujo sanguíneo a ese nivel[16].

En 1840 Guillaume Duchenne mapea la función muscular usando cargas eléctricas y más tarde, a finales de 1880 Sir Victor Horsley emplea la neuroestimulación a nivel intraoperatorio para identificar focos de activación en pacientes epilépticos[16].

Quizás la publicación más famosa fue la del Dr. Shealy en 1967, no solo por ser la primera experiencia directa en cuanto a un dispositivo para manejo de dolor cercano a la médula espinal en humanos, sino por el paciente en el que se empleó[18]. Su reporte de caso, toma como antecedente la serie de casos del mismo año expuesta con anterioridad por Wall y Sweet de 8 pacientes con dolor neuropático, quienes reportaron alivio del dolor por más de 1 hora tras estimulación eléctrica de nervios periféricos[5]. Shealy reporta un paciente de 70 años con cáncer broncogénico y metástasis a hígado y pleura, que curso con picos febriles y, además tenía una expectativa de vida de 1 a 2 meses al cual se le realizó laminectomía y suturó el dispositivo a duramadre para estimular cordones posteriores a nivel de T3, el paciente presentó muy buena respuesta del manejo del dolor, desafortunadamente desarrolló hemiplejia derecha muriendo a los pocos días por una endocarditis bacteriana[18].

A pesar de la breve experiencia con estos dispositivos, un año después Medtronic obtiene la aprobación de la FDA para comercializar sus dispositivos para el tratamiento del dolor. Muchos efectos adversos y complicaciones surgieron en el camino tales como: Fuga de líquido cefalorraquídeo, compresión de la médula espinal, rechazo del sistema, infección, dolor en la herida, así como disfunción del equipo como tal; todo esto hizo que, inicialmente, no se generalizara como terapia aceptada.

Con el tiempo se fue optimizando su uso, el mejor ejemplo es que en 1972 se crean electrodos cilíndricos flexibles que se podían colocar de manera percutánea. Además estructuras de platino o titanio, mejor agarre para disminuir la migración, dispositivos recargables que aumentan la vida útil (alrededor de 10 años) y recientemente cambios en la frecuencia de estimulación que ha evolucionado de tónica, estimulación en ráfagas y alta frecuencia que disminuyen las parestesias y hacen el sistema más confortable para el paciente, así como nuevos puntos de estimulación como los son el ganglio de raíz dorsal y dispositivos compatibles con resonancia magnética, entre otros[16]. La expansión de esta tecnología no cesa e incluso está abarcando otros campos ajenos al dolor. Se puede apreciar mejor dicha evolución en la Tabla 1.

\section{Mecanismo de acción}

Como se expuso anteriormente, aunque la teoría de la compuerta sigue siendo la base para explicar el efecto terapéutico, no logra abarcar del todo su mecanismo. Recientemente, se ha comprobado la existencia de neuronas gabaérgicas inhibitorias en la superficie del asta posterior en la lámina II que pueden ser activadas por impulsos de fibras A beta, lo cual comprueba la teoría de la compuerta (en ratones transgénicos homocigotos)[18]. También por estudios electrofisiológicos en ratas se demuestra que la electroestimulación de cordón posterior inhibe la respuesta de neuronas de amplio rango dinámico mediada por fibras $C$ en lesiones nerviosas.

Si bien es cierto la gran mayoría de resultados que explican los mecanismos de esta terapia son estudios en ratas, y de inyección de agonistas o antagonistas a nivel intratecal; los mecanismos en términos generales que lo explican[19] además de la teoría de la compuerta son:

- Impulsos ortodrómicos que activan tractos inhibitorios descendentes.

- Modulación del sistema nervioso simpático.

- Mecanismos de regulación hacia arriba y regulación hacia abajo de neuromoduladores y neurotransmisores.

Actualmente, se prefiere explicar los mecanismos basados en el nivel en que actúan, en ese sentido se 


\section{Tabla 1. Evolución de la neuroestimulación}

\begin{tabular}{ll}
\hline 1965 & Walls y Melzack, publican su teoría de la compuerta \\
1967 & Shealy, primer uso clínico de estimulación de cordones posteriores \\
1968 & Sweet y Wepsic, realizan estimulación periférica implantable \\
1972 & Bechtereva, realizan estimulación crónica de ganglios basales para Parkinson \\
1976 & Cook y Dooley, realizan neuroestimulación para espasticidad \\
1982 & Tasker, publica atlas de estimulación talámica \\
1985 & Augustinsson, realiza neuroestimulación para enfermedad vascular periférica \\
1987 & Murphy y Giles, emplean neuroestimulación para angina refractaria \\
1991 & Tsubokawa, realiza estimulación corteza motora para alivio del dolor \\
1993 & Barolat desarrolla mapa de estimulación de cordones posteriores \\
1999 & Melzack publica su teoría de la neuromatriz \\
2004 & Se crea generador de pulso implantable recargable \\
2013 & Se crea generador de pulso compatible con RMN \\
2015 & Se aprueba la terapia de alta frecuencia y de estimulación de ganglio de raíz dorsal \\
\hline
\end{tabular}

Modificado de[16].

pueden presentar a nivel local, cerebral y periférico[20].

A nivel local es quizás lo más conocido, se sugiere que esta terapia induce cambios neuroquímicos por segmentos en el asta posterior, los cuales atenúan la hiperexcitabilidad en neuronas de amplio rango dinámico. Específicamente hay evidencia que sustenta la liberación de GABA B, acetilcolina y serotonina así como la supresión de aminoácidos excitatorios como glutamato y aspartato.

Aunado a lo anterior y gracias a las tecnologías de tomografía por emisión de positrones y la resonancia magnética funcional se ha logrado comprobar la activación de ciertas áreas a nivel cerebral durante y después de la terapia de neuroestimulación medular, lo cual explicaría porqué el efecto perdura aún después de haber apagado el dispositivo. Dichos cambios se han identificado en el tálamo contralateral a la extremidad con dolor y la corteza de asociación parietal (bilateral), ambas regulan los umbrales de dolor; así como la corteza cingulada anterior y áreas prefrontales que controlan el aspecto emocional. En pacientes con síndrome poslaminectomía tratados con esta terapia se observa desactivación del circuito cerebelotalamocortical que actúa como integrador de impulsos aferentes de nocicepción[20]. Estas descripciones retoman el concepto de neuromatriz descrito años atrás por el Dr. Melzack.

Los efectos a nivel periférico se encargan de explicar sobre todo los procesos de vasodilatación y aumento del flujo sanguíneo para el tratamiento de desórdenes isquémicos dolorosos. Aunque no se comprende del todo, se atribuye a la mejoría en la microcirculación resultado tanto de la modulación inhibitoria del sistema simpático como de mecanismos de vasodilatación antidrómica que permite redistribución del flujo y disminución del dolor.

\section{Guías de práctica clínica}

Actualmente, existen muchas guías y consensos principalmente por dos motivos: El primero es que se trata de una tecnología en continua renovación por los nuevos usos que se le atribuyen tanto en el campo de la algología como en muchos otros; la segunda razón es porque, aunque se siguen realizando estudios, aún se desconocen muchos aspectos de la misma incluyendo su mecanismo en todas sus áreas de expansión.

Las primeras serían las guías europeas (E.F.N.S) ya que estas datan de 2007. En resumen, nos dicen que hay evidencia II $B$, tanto para síndrome poslaminectomía como para síndrome doloroso regional complejo tipo I; para el resto de patologías describe evidencia IV D (síndrome doloroso regional complejo tipo II, lesión de nervio periférico, neuropatía diabética, neuralgia posherpética, lesión o avulsión de plexo braquial, dolor de muñón y miembro fantasma, dolor facial y dolor central), además, no se encuentra evidencia en ese 
momento para síndrome de cuello fallido[21].

Posteriormente, los británicos, que a diferencia de las anteriores lo establecen según el grado de respuesta[23]. En ese caso serían una buena indicación porque presenta buena respuesta el síndrome poslaminectomía, angina refractaria, dolor neuropático secundario a lesión parcial de nervio periférico y dolor radicular tras cirugía de columna cervical. Con respuesta intermedia están: dolor asociado a enfermedad vascular periférica, neuralgia intercostal y síndrome postoracotomía así como síndrome doloroso regional complejo. Con pobre respuesta y por lo tanto no indicado, la avulsión de plexo braquial, dolor axial nociceptivo posterior a cirugía, dolor central no medular, dolor por lesión medular, neuralgia posherpética y dolor de miembro fantasma. Asimismo, está el instituto de excelencia clínica (N.I.C.E) quienes recomiendan su utilización en dolor crónico de origen neuropático no isquémico de intensidad moderada a severa y la implementación de un test positivo valorando el coste y la duración del siste$\mathrm{ma}[23]$.

Las guías sudafricanas son muy parecidas a las británicas, solo agregan un nivel más que sería sin respuesta en casos como sección completa de médula espinal, dolor nociceptivo no isquémico y avulsión de raíz nerviosa[24].

Los españoles por su parte, junto con la sociedad europea de anestesia regional (E.S.R.A), publican sus propias guías, en donde en uno de sus capítulos se habla más específicamente de criterios de inclusión y exclusión. Dentro de los criterios de inclusión se mencionan[26]:

- Dolor refractario a tratamientos convencionales.

- No tener indicación quirúrgica.

- Presencia de dolor neuropático, no maligno preferentemente.

- Valoración por psicólogo/psiquiatra con formación en dolor que confirme perfil psicológico adecuado.

- No padecer otras patologías crónicas graves.

- No historia de consumo de drogas o alcohol.

- No tener problemas legales (estudio de cada caso).

- Período de prueba eficaz para el dolor, previo al implante definitivo, a excepción del paciente anginoso que se realizará la técnica en un solo tiempo.

- Imprescindible conseguir cobertura de la zona dolorosa con la parestesia.

Se excluyen aquellos pacientes con:

- Inestabilidad psicológica.

- Historia de abuso de alcohol y drogas.
- Coagulopatías severas.

- Falla en la prueba de neuroestimulación.

- Cirugía pendiente.

- Inadecuado tratamiento previo.

- Pobre red de apoyo.

- Incapacidad para comprender el funcionamiento del sistema.

La Sociedad Internacional de Neuromodulación hace en el año 2014 una evaluación crítica de las guías disponibles hasta ese momento, concluyendo que se debe seguir generando evidencia, recomendando la realización de mejores estudios[26].

\section{Indicaciones y selección de pacientes}

Tomando en cuenta las recomendaciones anteriores se pueden establecer lineamientos básicos acerca de las indicaciones. Muchos autores sugieren que la neuromodulación se debe considerar en ciertos tipos de dolor neuropático e isquémico, después que la terapia convencional falle[2].

Aunque no hay indicaciones claras en cuanto a evidencia fuerte, se pueden mencionar las siguientes como indicaciones claras descritas en todas las guías: la patología con más evidencia de adecuada respuesta sigue siendo el síndrome poslaminectomía con una eficacia significativa nivel I a II[27], sin embargo, se pueden agregar otras como el síndrome doloroso regional complejo (sobre todo tipo I) y condiciones dolorosas de tipo isquémico como claudicación de miembros inferiores por enfermedad arterial periférica junto con la angina refractaria[19].

Al respecto Atkinson[28], hace una revisión extensa sobre recomendaciones para identificar la población que se puede beneficiar de forma óptima, incluso aporta un algoritmo que toma en cuenta sobre todo la valoración multidisciplinaria (incluyendo psicología) del paciente que ha cumplido criterios de inclusión, como el test de prueba. Básicamente el algoritmo consiste en:

- Definir el dolor como crónico, con una adecuada historia clínica, examen físico e imágenes para definir un síndrome doloroso.

- Si el paciente no responde a tratamiento convencional, enviarlo a un centro de dolor multidisciplinario en donde se ajusten los medicamentos y se aborde nuevamente el diagnóstico.

- En caso de que con un diagnóstico fidedigno y un tratamiento óptimo el paciente siga con pobre respuesta, se debe someter a evaluación para ver si cumple criterios de inclusión y no presenta con- 
traindicaciones de ningún tipo (evaluación biopsicosocial).

- Si el paciente pasa la evaluación se somete a un test de prueba con un dispositivo temporal externo (en donde manifieste disminución de la intensidad del dolor de al menos 50\%, disminución del consumo de analgésicos, mejoría en calidad del sueño o calidad de vida).

- Si pasa el test de prueba se coloca el dispositivo, no sin antes establecer metas de alivio entre médico-paciente, la duración y el seguimiento.

Se requiere de un equipo médico con entrenamiento en la técnica y la presencia del técnico del equipo para asegurar la colocación óptima del dispositivo, tanto en la fase de prueba como en la definitiva. En general, la posición definitiva de los electrodos en el espacio epidural suele localizarse entre C4 a C7 para dolores que afecten a la zona cervical y extremidades superiores, y entre T8 y T11 en dolores axiales de la zona lumbosacra y de extremidades inferiores. Los parámetros eléctricos que permiten los equipos son 0 a 10,5 V de intensidad de corriente, 2 a $130 \mathrm{~Hz}$ de frecuencia y de 60 a $450 \mu$ seg de ancho de pulso.

Pueden funcionar en modalidad continua o cíclica aunque, recientemente, algunos equipos han incorporado gamas más altas de frecuencias de hasta 10 $\mathrm{KHz}[29]$.

\section{Más allá del dolor}

Lo expuesto a lo largo de este artículo tiene como objetivo exponer el uso de la neuromodulación en el campo de la medicina del dolor y más específicamente a nivel espinal, sin embargo, como se comentó antes la neuromodulación tiene un enorme potencial de aplicaciones clínicas en psiquiatría, en neurología y neurorrehabilitación, en la adicción entre otras, además aquí tampoco se está tomando en cuenta otras modalidades de neuromodulación como lo son la estimulación cerebral profunda, estimulación de nervios periféricos, estimulación del ganglio de raíz dorsal, estimulación de corteza motora y la estimulación magnética transcraneal.
Lo más reciente en el campo de la neuromodulación es lo que se denomina expansión de las capacidades humanas o mejoramiento de funciones cognitivas y sensoriales, es decir, no solo en individuos con patología, sino en individuos sanos, este es un campo que llama incluso a la intervención de la bioética sin detener por eso su expansión.

En ese sentido, el mejoramiento cognitivo se puede definir como la amplificación o extensión de las capacidades centrales de la mente al aumentar el sistema de procesamiento de la información. Definiendo cognición como el proceso de organizar la información, lo cual va desde la adquisición de información, selección, comunicación, representación y retención que involucra mecanismos de sensación y percepción, atención, lenguaje y memoria, y repercute en el razonamiento y coordinación de impulsos motores. Todas estas investigaciones se dan gracias a dispositivos no invasivos (estimulación magnética transcraneal, estimulación de corriente directa transcraneal y estimulación de corriente alterna transcraneal)[20].

Específicamente, en el campo del procesamiento de lenguaje, se ha visto que la estimulación de corriente directa transcraneal de la corteza prefrontal y la región perisilviana posterior (específicamente el área de Wernicke y el giro temporal superior) en individuos sanos mejora la afluencia verbal[20].

En el área cognitiva tanto en la memoria de trabajo como en la inhibición de respuesta (la cual está alterada en pacientes con trastorno de hiperactividad y déficit atencional), tras estimulación de corriente directa transcraneal anodal, se presenta aumento en el rendimiento que podía perdurar semanas después de dicho estimulo[20].

En conclusión, el uso clínico de la neuromodulación sigue creciendo. Posiblemente, no hemos alcanzado a tocar la superficie de la capacidad terapéutica de este grupo de herramientas; aunque en este momento la aplicación más difundida y mejor establecida sigue siendo el tratamiento del dolor. De lo anterior es necesario comprender bien los mecanismos fundamentales e indicaciones principales que justifiquen el uso de esta valiosa herramienta en desarrollo para aplicarlo al grupo de pacientes que más se pueda beneficiar del mismo. 


\section{Referencias}

1. Kaku M. The Future of the Mind: The Scientific Quest to Understand, Enhance, and Empower the Mind. New York: Doubleday; 2014. pp. 12-23.

2. Florez J. Medicina dolor: Perspectiva internacional. Barcelona, España: ELSEVIER; 2015. pp. 582-90.

3. Acevedo J. Ronald Melzack and Patrick Wall. La teoría de la compuerta. Rev Soc Esp Dolor. 2013;20(4):191-202.

4. Melzack R, Wall PD. On the nature of cutaneous sensory mechanisms. Brain. 1962 Jun;85(2):331-56. https://doi. org/10.1093/brain/85.2.331 PMID:14472486

5. Wall PD, Sweet WH. Temporary abolition of pain in man. Science. 1967 Jan;155(3758):108-9. https://doi.org/10.1126/science.155.3758.108 PMID:6015561

6. Cooper SJ, Donald O. Donald O. Hebb's synapse and learning rule: a history and commentary. Neurosci Biobehav Rev. 2005 Jan;28(8):851-74. https://doi.org/10.1016/j. neubiorev.2004.09.009 PMID:15642626

7. Melzack R. The McGill Pain Questionnaire: major properties and scoring methods. Pain. 1975 Sep;1(3):277-99. https://doi.org/10.1016/03043959(75)90044-5 PMID:1235985

8. Serrano. Valoración del dolor. Rev Soc Esp Dolor. 2002;9:109121.

9. Melzack R. From the gate to the neuromatrix. Pain. 1999 Aug;6 Suppl 6:S1216. https://doi.org/10.1016/ S0304-3959(99)00145-1 PMID:10491980

10. Melzack R, Wall PD. Pain mechanisms: a new theory. Science. 1965 Nov;150(3699):971-9. https://doi.org/10.1126/science.150.3699.971 PMID:5320816

11. Moayedi M, Davis KD. Theories of pain: from specificity to gate control. J Neurophysiol. 2013 Jan;109(1):5-12. https://doi. org/10.1152/jn.00457.2012 PMID:23034364

12. Kreis P. Spinal Cord Stimulation. Percutaneous implantation techniques. New York, USA: OXFORD University Press; 2009. pp. 210-21.

13. Melzack R. From the gate to the neuromatrix. Pain. 1999 Aug;6 Suppl 6:S1216. https://doi.org/10.1016/ S0304-3959(99)00145-1 PMID:10491980

14. Melzack R. Phantom limbs, the self and the brain. Can Psychol. 1989;30(1):1-16. https://doi. org/10.1037/h0079793.

15. Melzack R. Evolution of the neuromatrix theory of pain. The Prithvi Raj Lecture: presented at the third World Congress of World Institute of Pain, Barcelona 2004. Pain Pract. 2005 Jun;5(2):85-94. https://doi.org/10.1111/ j.1533-2500.2005.05203.x PMID:17177754

16. Iannetti GD, Mouraux A. From the neuromatrix to the pain matrix (and back). Exp Brain Res. 2010 Aug;205(1):1-12. https:// doi.org/10.1007/s00221-0102340-1 PMID:20607220

17. Deer T. Treatment of Chronic Pain by Interventional Approaches AMERICAN ACADEMY of PAIN MEDICINE Textbook on Patient Management. New York, USA: Springer; 2015. pp. 212-50

18. Shealy CN, Mortimer JT, Reswick $J B$. Electrical inhibition of pain by stimulation of the dorsal columns: preliminary clinical report. Anesth Analg. 1967 Jul-Aug;46(4):489-91. https:// doi.org/10.1213/00000539196707000-00025
PMID:4952225

19. Daniele CA, MacDermott AB Low-threshold primary afferent drive onto GABAergic interneurons in the superficial dorsal horn of the mouse. J Neurosci. 2009 Jan;29(3):68695. https://doi.org/10.1523/ JNEUROSCI.5120-08.2009 PMID:19158295

20. Guan Y. Spinal cord stimulation: neurophysiological and neurochemical mechanisms of action. Curr Pain Headache Rep. 2012 Jun;16(3):217-25. https://doi. org/10.1007/s11916-012-02604 PMID:22399391

21. Knotkova H. Textbook of Neurostimulation. Principles, Methods and Clinical Applications USA. New York: Springer; 2015. pp. 187-275.

22. European Federation of Neurological Societies guidelines on neurostimulation therapy for neuropathic pain. Eur J Neurol. 2007;14:952-70. https://doi.org/10.1111/j.14681331.2007.01916.x.

23. British Pain Society. Spinal cord stimulation for the management of pain: recommendations or best clinical practice; 2009. Disponible en : https://www.britishpainsociety.org/static/uploads/ resources/files/book

24. National Institute for Health and Clinical Excellence. Spinal cord stimulation for chronic pain of neuropathic or ischemic origin; 2008. Disponible en: https:// www.nice.org.uk/guidance/ ta159

25. Raff M, Melvill R, Coetzee G, Smuts J, Pain SA, Pain SA. Spinal cord stimulation for the management of pain: recommendations for best clinical practice. S Afr Med J. 2013 Mar;103(6 Pt 2 Suppl):423-30. https:// doi.org/10.7196/SAMJ.6323 PMID:23725965

26. Sociedad Europea de Aneste- 
sia Regional y Tratamiento del dolor Asociación delegada en España. Grupo español de neuromodulación. Guía española de neuroestimulación en el dolor crónico; Spanish guidelines for neurostimulation; Spanish Group of Neuromodulation. España, Valencia. ESRA. 2005. p 19-25

27. Deer TR, Thomson S, Pope JE, Russo M, Luscombe F, Levy R. International neuromodulation society critical assessment: guideline review of implan- table neurostimulation devices. Neuromodulation. 2014 Oct;17(7):678-85. https:// doi.org/10.1111/ner.12186 PMID:24802237

28. Grider JS, Manchikanti L, Carayannopoulos $A$, Sharma $M L$, Balog CC, Harned ME, et al. Effectiveness of Spinal Cord Stimulation in Chronic Spinal Pain: A Systematic Review. Pain Physician. 2016 Jan; 19(1):E33-54. PMID:26752493

29. Atkinson L, Sundaraj SR, Brooker
C, O'Callaghan J, Teddy P, Salmon J, et al. Recommendations for patient selection in spinal cord stimulation. J Clin Neurosci. 2011 Oct;18(10):1295302. https://doi.org/10.1016/j. jocn.2011.02.025 PMID:21719293

30. García-March G, García-Catalán G. Neuromodulacion eléctrica y dolor raquídeo. Rev Soc Esp Dolor. 2015;22(3):116-25. https://doi.org/10.4321/S113480462015000300006 